\title{
Comparison of Metal Oxide Catalysts for Pyrolytic MOLI-MS Bacterial Identification
}

\author{
Kent J. Voorhees ${ }^{1}{ }^{*}$, Nicholas R. Saichek ${ }^{1}$, Kirk R. Jensen ${ }^{1}$, Peter B. Harrington ${ }^{2}$ and \\ Christopher R. Cox ${ }^{1}$ \\ ${ }^{1}$ Department of Chemistry, Colorado School of Mines, Golden, CO 80401 \\ ${ }^{2}$ Center for Intelligent Chemical Instrumentation, Department of Chemistry \& \\ Biochemistry, Ohio University, Athens, OH, 45701
}

\begin{abstract}
Six metal oxide catalysts were investigated for effective cleavage and laser ionization of bacterial cell wall phospholipid extracts. Calcium oxide has been utilized in the past for this application, but suffered from degradation of catalytic activity. Following evaluation of the six catalysts, $\mathrm{CeO}_{2}$ was found to be the most stable over time and produced fatty acid profiles of bacterial pathogens similar to freshly activated $\mathrm{CaO}$. A suite of ten bacteria previously studied using $\mathrm{CaO}$ catalysis provided cross validation results of analysis of $97 \%$ correct correlation for negative-ion data. Cerium oxide catalysis studies yielded cross validation results of $100 \%$. Using analysis of variance-principal component analysis (ANOVA-PCA), four sample sets collected with stored catalyst at $0,8,24$, and $504 \mathrm{~h}$ showed no effect based on long-term catalyst degradation. Supervised learning by a fuzzy rule-building expert system (FuRES) that was validated with training and prediction set partitions independent of $\mathrm{CeO}_{2}$ age and unsupervised data analysis using a dendrogram of Euclidean distance confirmed that the $\mathrm{CeO}_{2}$ catalyst age had no effect on the fatty acid mass spectral profiles. Key words: MALDI-MS, MOLI-MS catalytic pyrolysis, chemometrics, bacteria, fatty acids
\end{abstract}




\section{Introduction}

Fatty acid profiling has been used for bacterial identification since the 1960s [1]. The original work utilized an ex situ method involving saponification followed by derivatization of cell wall fatty acids to form fatty acid methyl esters (FAMEs) followed by gas chromatography analysis [2]. Later, an in situ thermochemical process using tetramethyl ammonium hydroxide (TMAH) was developed, which significantly reduced sample analysis time to less than three min for Curie-point pyrolysis-mass spectrometry (PyMS) [3,4].

It has been observed during lipid analysis by matrix-assisted laser desorption ionization time-of-flight mass spectrometry (MALDI-TOF MS), that sufficient heat was generated to pyrolyze the sample [5]. During an investigation of TMAH as a methylating agent for MALDI-TOF MS with $\mathrm{CaO}$ as a matrix, lipid cleavage was observed. Tandem MALDI analysis identified the resulting cleavage products as calcium fatty acid adducts [5]. It was later determined that a strong base, acid, or heat was necessary to activate $\mathrm{CaO}[5,6]$. Using activated $\mathrm{CaO}$, monoacylglycerides, diacylglycerides, triacylglycerides, phospholipids, as well as bacterial and algal extracts, produced peaks for their constituent fatty acids [7]. In addition, spectra from bacterial and algal extracts were found to be reproducible, which suggested that this technique could be used for microbial fatty acid profiling. In a follow up publication, five Gram-positive and five Gram-negative bacteria were analyzed using $\mathrm{CaO}$ in positive- and negative-ion MALDITOF MS modes followed by principal component analysis (PCA) [8]. PCA showed distinct clusters for five replicates of each bacterial species with $94 \%$ cross validation 
correlation of positive-ion data. Negative-ion data yielded a higher classification rate of 97\% and exhibited less within group variation than the positive-ion data.

The current state-of-the-art for MALDI MS-based clinical microbial identification centers on protein profiling. Three commercial protein databases [9] have been developed for this application [10]. Two companies, Bruker and BioMérieux, have received European 'CE' mark and US Food and Drug Administration approval for use in clinical laboratories. These databases contain multiple profiles of over 3000 bacteria and claim over 98\% correct identification according to company literature. In an analysis of 1,371 clinical isolates, a peer-reviewed comparison of the Bruker Biotyper to conventional typing methods found $93.2 \%$ were putatively identified to the species level [11]. A second study focused specifically on Gram positive rods, which typically have lower Biotyper identification rates compared to Gram negatives or Gram positive cocci [12], reported $79.1 \%$ of 215 isolates were correctly identified at the species level [13]. The most important criterion for a diagnostic technique centers on the fact that each bacterial protein profile is unique and reproducible, which depends on bacterial growth conditions and instrument stability [14]. In the case of a catalytic process such as metal oxide lipid cleavage, catalyst stability must also be considered. The following paper describes the degradation associated with $\mathrm{CaO}$ stability and the screening of five additional metal oxide catalysts.

\section{Experimental}

\subsection{Bacterial strains and culture conditions}

Table 1 summarizes the bacteria used in this study [8]. All bacterial strains were cultured overnight in brain heart infusion (BHI) medium (BD-Difco, Franklin Lakes, NJ) at $37^{\circ} \mathrm{C}$ 
[8]. Following incubation of broth cultures, bacteria were streaked onto agar plates and incubated for $22 \mathrm{~h}$. Extracts of single colonies were immediately prepared upon removal from the incubator. To minimize culture conditions as a variable in fatty acid analysis, these parameters were used as a carefully controlled, standardized method for all experiments.

\subsection{Lipid extraction}

Individual colonies were suspended in $100 \mu \mathrm{L}$ of a $33 / 66 \mathrm{v} / \mathrm{v} \%$ methanol/chloroform mixture (Pharmco-AAPER, Shelbyville, KY and Fisher, Pittsburgh PA, respectively) and vortexed for $2 \mathrm{~min}$ at $3200 \mathrm{rpm}$. Phase separation was achieved by addition of $100 \mu \mathrm{L}$ of phosphate buffer saline (PBS, pH 7.4) followed by an additional $60 \mathrm{~s}$ of vortexing and cell debris removal by centrifugation for $30 \mathrm{~s}$ at $1400 \mathrm{x}$ g. The resulting organic fraction was then removed for MALDI-TOF MS analysis.

\subsection{Catalysts}

The following metal oxides were investigated for their combined catalytic properties and temporal stability during bacterial phospholipid cleavage: calcium oxide. purity $99.4 \%$ (Nano-Active Inc. Manhattan KS), magnesium oxide, purity 99.6\% (Nano-Active Inc,), nickel oxide, purity 99.8\% (Sigma Aldrich, St. Louis, MO), cerium (IV) oxide, purity 99.9\% (Cerac, Milwaukee,WI), barium oxide, purity 99.5\% (Cerac), and strontium oxide, purity 99.5\% (Alfa Aesar, Ward Hill, MA). Thermal activation was performed on $\mathrm{CaO}$, $\mathrm{BaO}$, and $\mathrm{SrO}$ in an open-air tube furnace at $800^{\circ} \mathrm{C}$ for $3 \mathrm{~h}$ while $\mathrm{NiO}$ and $\mathrm{MgO}$ were heated to $350{ }^{\circ} \mathrm{C}$ to remove surface water. Activation of $\mathrm{CeO}_{2}$ was not required. All catalysts were stored in a desiccator under vacuum.

An investigation of the stability of the $\mathrm{CaO}, \mathrm{BaO}$, and $\mathrm{SrO}$ was conducted by taking MALDI-TOF MS measurements termed metal oxide laser ionization-MS (MOLI-MS) of 
the same bacterial phospholipid extracts at 0,8 , and $24 \mathrm{~h}$ post activation. Cerium oxide spectra were generated at $0,8,24$, and $504 \mathrm{~h}$, where the catalyst for $\mathrm{t}=0$ was obtained directly from the manufacturer's bottle. This same catalyst was stored in a vacuum dessicator and removed for the 8, 24, and $504 \mathrm{~h}$ time points.

\subsection{Mass spectrometry}

Samples were prepared for MOLI-MS as previously described [7]. Briefly, $100 \mathrm{mg}$ catalyst was added to one $\mathrm{mL}$ of $n$-hexane (Sigma-Aldrich). One $\mu \mathrm{L}$ was then removed from the bottom of the resulting slurry and spotted on a stainless steel MALDI sample plate followed by $2 \mu \mathrm{L}$ of lipid extract. Other sample preparation methods such as overlay and sandwich methods, did not improve the data quality.

Mass spectrometric measurements were acquired in positive- and negative-ion modes with a Bruker Ultraflextreme MALDI-TOF mass spectrometer (Bruker Daltonics, Billerica, MA) equipped with a $355 \mathrm{~nm} \mathrm{Nd:YAG} \mathrm{laser.} \mathrm{Spectra} \mathrm{were} \mathrm{collected} \mathrm{in}$ reflector mode at 4000 resolution with a grid voltage of $50.3 \%$, a delayed extraction time of $120 \mathrm{~ns}$, and a low-mass cutoff of $150 \mathrm{Da}$. Five replicate spectra were collected for each bacterium as 500 shot composites at a sampling frequency of $1 \mathrm{kHz}$ using automated laser rastering.

\subsection{Data analysis}

Selected fatty peaks (Table 2) from each spectrum were exported from the instrument in ASCII format and processed by two procedures. In the first, an in-house program centroided and normalized the spectra to total intensity, and selected fatty acid peaks prior to PCA. Processed data were recorded in a tab-delimited format and imported directly into R Version 3.0.2 (The R Foundation for Statistical Computing, Vienna, 
Austria) as a data frame. PCA was performed using the $\operatorname{prcomp}()$ function with an argument passed to return rotated variables; data were automatically mean-centered by $\operatorname{prcomp}()$. Data were plotted using the built-in plot() function in R. Leave-one-out cross validation was performed on the input data by passing the appropriate argument to the linear discriminate analysis function $l d a()$, which is part of the Modern Applied Statistics with S package [15]. Results from $l d a()$ returned class assignment based on supervised learning.

In supervised analysis, the data were imported into MATLAB R14a using the 150 unprocessed spectra as text files. The spectra were normalized to unit vector length and analyzed using a fuzzy rule-building expert system (FuRES) [16] with customized MATLAB scripts and functions. The FuRES was validated with 100 bootstraps with 3 Latin partitions $[17,18]$. The data were bootstrapped individually and also by removing all the replicates that corresponded to the same catalyst age. The normalized data were also used to construct an unsupervised dendrogram using average centroid linkages and minimum Euclidean distances. The normalized mass spectra also were subjected to analysis of variance-principal components analysis (ANOVA-PCA) with bacterial species, catalyst age, and interaction as factors. The statistical significance was measured by using the pooled sums of squares method that provides a conservative estimate of significance.

\section{Results and Discussion}

MOLI-MS using $\mathrm{CaO}$ as a catalyst is known to cleave phospholipids into their fatty acids [5,7]. Although cross-validation results were above $97 \%$ correct classification for the previously referenced 10 bacteria, for the technique to be useful in the clinical laboratory, 
the catalyst must be stable over time and capable of producing highly reproducible fatty acid profiles. $\mathrm{CaO}$ is known to be susceptible to moisture and carbon dioxide poisoning and must be activated prior to use for catalytic pyrolysis of lipids. It was unclear if the catalyst could be stored under vacuum and maintain its original activity. Fig. 1 is a PCA score plot of the first 2 principal components (PCs) for negative-ion CaO-catalyzed MOLI mass spectra of Escherichia coli, Salmonella typhimurium, and Shigella boydii extracts. Measurements of a single extract from each bacterial type were taken over three time points from 0,8 , and $24 \mathrm{~h}$ post-thermal catalytic activation. Although unique spatial groupings corresponding to each time point were observed, the relative position of distinct groupings for each bacterial species changed with time, and clearly showed a temporal effect on catalyst deactivation. The overlapping alignment observed for the $S$. boydii and S. typhimurium groupings suggests that these genera may be difficult to differentiate using supervised learning techniques. Table 3 summarizes the variance associated with the first six PCs. Group separation was observed at a cumulative variance of $85.6 \%$ using PCs 1 and 2 .

Because $\mathrm{CaO}$ appears to be deactivated even in short time periods, five additional prospective catalysts were evaluated for generation of fatty acids from glyceride standards and their overall stability investigated over a 24-504 h timeframe. Nickel oxide and $\mathrm{MgO}$ failed to cleave glyceride standards to any degree regardless of instrument parameters, rather, molecular species of intact glycerides in both positive- and negativeion modes were observed. Cerium oxide, $\mathrm{BaO}$, and $\mathrm{SrO}$ cleaved the standards (data not shown) and $\mathrm{BaO}$ and $\mathrm{SrO}$ produced both positive- and negative-ion peaks in the same manner as previously reported for $\mathrm{CaO}$. However, only negative-ions were observed for 
$\mathrm{CeO}_{2}$. This result was not surprising because the +4 oxidation state of cerium produces a +3 charge on resulting single fatty acid complexes. The low-mass, non-integer peaks of these complexes places them in a spectral region that complicates data analysis. Based on reactivity and adverse health effects, $\mathrm{BaO}$ and $\mathrm{SrO}$ were not investigated beyond our initial screening. Because it's observed reactivity, $\mathrm{CeO}_{2}$ was subjected to further in-depth studies.

To determine whether $\mathrm{CeO}_{2}$ demonstrated improved stability over $\mathrm{CaO}$, a time study was conducted with the same 10 bacteria listed in Table 1. Fig. 2 contains selected negative ion spectra of Salmonella from 0, 8, and $24 \mathrm{~h}$ for the two catalysts. The intensities of the three spectra in Fig. $2 \mathrm{~B}$ obtained using $\mathrm{CeO}_{2}$ were consistent with each other as a function of time and comparable to the $\mathrm{CaO}$-derived spectrum at time zero in Fig. 2A. Calcium oxide spectra (Fig. 2B) obtained at 8 and $24 \mathrm{~h}$ showed changes in intensity of the individual fatty acids and a prominent, unidentified peak at $m / z 473$, indicating degradation of the catalyst. Fig. 3 shows PCA score plots of $\mathrm{CeO}_{2}$ derived bacterial data and previously published $\mathrm{CaO}$ data for the same bacteria [8]. Table 4 summarizes the variance associated with the first six PCs for each catalyst. The cumulative variance for the $\mathrm{CeO}_{2}$ data was $92.1 \%$ for the first $2 \mathrm{PCs}$. In contrast, the cumulative variance for $\mathrm{CaO}$ was only $73 \%$ encompassing $6 \mathrm{PCs}$. In the case of the $\mathrm{CaO}$ data, all combinations (PCs 1 and 2, PCs 1 and 3, etc.) were plotted, however, PCs 1 and 2 gave the best separation. Comparison of 3D plots of the first $3 \mathrm{PCs}$ for $\mathrm{CaO}$ (Fig. 3A) and $\mathrm{CeO}_{2}$ (Fig. 3B) had similar separations (between group variation), however the precision (within group variation) was better using $\mathrm{CeO}_{2}$. Cross-validation using LDA of $\mathrm{CeO}_{2}$-derived fatty acid profiles gave $100 \%$ correct classification. 
Analysis of variance-principal components analysis [19] was used to determine the significant factors responsible for differentiation. The $p$-value for the mathematical means between species was below $10^{-4}$. The storage time of $\mathrm{CeO}_{2}$ and interaction among species factors were highly insignificant with $p$-values close to 1.0. [19]

Cluster analysis of the data produced the dendrogram of the Euclidean distances between pairs of objects and the cluster averages represented in Fig. 4. It is interesting that two distinct clusters were observed that almost corresponds to Gram type, which was previously observed by FAME analysis using PyMS [20]. If E. faecalis and F. tularensis were interchanged, separation of Gram type would be complete. Note that the separation that occurs among the species in the dendrogram is natural, and not because of unsupervised clustering of the data.

A principal component transform (PCT) was used to compress the spectra before FuRES analysis. The classification tree in Fig. 5 is similar in structure to the dendrogram in Fig. 4, although FuRES [19] is supervised and the cluster analysis was unsupervised. For the PCT during the bootstrap procedures, principal components were calculated from the training data set and prediction data were projected onto these components for each bootstrap-partition.

The results were $100 \%$ accurate for classification at the species level. Further analysis used a partition procedure so that each group of spectra collected for a specific catalyst age was removed from the model-building step using the same procedure as before of 100 bootstraps and 3 partitions. Replicate spectra from the same catalyst age and species were either in the prediction set or training set, but never both at the same time. 
Classification accuracy was not affected by $\mathrm{CeO}_{2}$ post-activation time and remained at $100 \%$.

\section{Conclusions}

In total, six metal oxide catalysts were evaluated for their capacity to reproducibly cleave bacterial cell wall phospholipid extracts to their constituent fatty acids followed by negative-ion MOLI MS. $\mathrm{CaO}$ was found to partially degrade in less than one-hour postactivation. Of the six catalysts evaluated, $\mathrm{CeO}_{2}$ provided superior stability and reproducibility and required no activation. An extended time study up to $504 \mathrm{~h}$ using the same fatty acid extract and stored catalyst showed that no difference in fatty acid distribution occurred. A comparison of $\mathrm{CaO}$ data collected immediately after activation to $\mathrm{CeO}_{2}$-derived data collected over a one-week period showed that similar fatty acid profiles were observed and clearly established the stability of the $\mathrm{CeO}_{2}$. Both supervised and unsupervised classifiers gave 100\% accurate species assignment, demonstrating the future potential as a clinical technique to enhance current MALDI protein profiling.

\section{Acknowledgments.}

The authors acknowledge a Career Development Award to C.R.C from the Rocky Mountain Regional Center of Excellence for Biodefense and Emerging Infectious Diseases Research supported by National Institute of Allergy and Infectious Diseases grant U54 AI065357 and the Colorado School of Mines Department of Chemistry for support of K.R.J. Acquisition of the MALDI-TOF mass spectrometer was supported in part by National Science Foundation MRI grant CHE-1229156. 


\section{Literature cited.}

[1] K. Abel, H. Deschmertzing and J.I. Peterson, Characterization of Bacteria by Gas Chromatography: Comparison of Trimethylsilyl Derivatives of Whole-Cell Hydrolysates, J Bacteriol, 85, (1963) 1039.

[2] L.J. Raines, C.W. Moss, D. Farshtchi and B. Pittman, Fatty acids of Listeria moncytogenes, J Bacteriol, 96, (1968) 2175.

[3] G. Holzer, T.F. Bourne and W. Bertsch, Analysis of in situ methylated microbial fatty acid constituents by Curie-point pyrolysis-gas chromatography-mass spectrometry, J. Chromatog, 468, (1989) 181.

[4] F. Basile, M.B. Beverly, C. Abbas-Hawks, C.D. Mowry, K.J. Voorhees and T.L. Hadfield, Direct mass spectrometric analysis of in situ thermally hydrolyzed and methylated lipids from whole bacterial cells, Anal Chem, 70, (1998) 1555.

[5] K.J. Voorhees, C.R. McAlpin and C.R. Cox, Lipid Profiling Using Catalytic Pyrolysis/ Metal Oxide Laser Ionization-Mass Spectrometry, J. Anal. Appl. Pyrol., 98, (2012) 201.

[6] R.M. Morris and K.J. Klabunde, Formation of paramagnetic adsorbed molecules on thermally activated magnesium and calcium oxides. Further studies of carbon monoxide, J. Am. Chem. Soc., 105, (1983) 2633.

[7] C.R. McAlpin, K.J. Voorhees, A.R. Corpuz and R.M. Richards, Analysis of Lipids: Metal Oxide Laser Ionization Mass Spectrometry, Anal Chem, 84, (2012) 7677.

[8] K.J. Voorhees, K.R. Jensen, C.R. McAlpin, J.C. Rees, R. Cody, M. Ubukata and C.R. Cox, Modified MALDI MS Lipid Profiling for Identification of Bacteria, $J$ Mass Spectrom, 48, (2013) 850.

[9] D. Martiny, L. Busson, I. Wybo, R.A. El Haj, A. Dediste and O. Vandenberg, Comparison of the Microflex LT and Vitek MS systems for routine identification of bacteria by matrix-assisted laser desorption ionization-time of flight mass spectrometry, J Clin Microbiol, 50, (2012) 1313.

[10] R.D. Holland, J.G. Wilkes, F. Rafii, J.B. Sutherland, C.C. Persons, K.J. Voorhees and J.O. Lay, Jr., Rapid identification of intact whole bacteria based on spectral patterns using matrix-assisted laser desorption/ionization with time-of-flight mass spectrometry, Rapid Commun Mass Spectrom, 10, (1996) 1227. 
[11] A. Bizzini, C. Durussel, J. Bille, G. Greub and G. Prod'hom, Performance of Matrix-Assisted Laser Desorption Ionization-Time of Flight Mass Spectrometry for Identification of Bacterial Strains Routinely Isolated in a Clinical Microbiology Laboratory, Journal of Clinical Microbiology, 48, (2010) 1549.

[12] B. Schulthess, K. Brodner, G.V. Bloemberg, R. Zbinden, E.C. Bottger and M. Hombach, Identification of Gram-positive cocci using MALDI-TOF MS: comparison of different preparation methods and implementation of a practical algorithm for routine diagnostics, J Clin Microbiol, 51, (2013) 1834.

13] B. Schulthess, G.V. Bloemberg, R. Zbinden, E.C. Bottger and M. Hombach, Evaluation of the Bruker MALDI Biotyper for identification of Gram-positive rods: development of a diagnostic algorithm for the clinical laboratory, J Clin Microbiol, 52, (2014) 1089.

[14] C. Kunitsky, G. Osterhout and M. Sasser, in M. Miller (Ed.), Encyclopedia of Rapid Microbiological Methods, Davis Healthcare International Publishing Moore, OK, 2013, Chapter 1, p. 1.

[15] W.N. Venables and B.D. Ripley, Modern Applied Statistics with S., Springer, New York, N.Y., 2002.

[16] P.B. Harrington, Fuzzy Rule-Building Expert Systems: Minimal Neural Network Journal of Chemometr, 5, (1991) 467.

[17] P.B. Harrington, Application of Linear and Nonlinear Discrete ... Bootstrapped Latin Partitions, Trends in Anal Chem, 25, (2006) 1112.

[18] C.H. Wan and P.D. Harrington, Screening GC-MS data for carbamate pesticides with temperature-constrained-cascade correlation neural networks, Anal Chim Acta, 408, (2000) 1.

[19] P.D. Harrington, N.E. Vieira, J. Espinoza, J.K. Nien, R. Romero and A.L. Yergey, Proteomic analysis of amniotic fluids using analysis of variance principal component analysis and fuzzy rule-building expert systems applied to matrixassisted laser desorption/ionization mass spectrometry, Anal Chim Acta, 544, (2005) 118.

[20] F. Basile, K.J. Voorhees and T.L. Hadfield, Microorganism Gram-Type Differentiation Based on Pyrolysis-Mass Spectrometry of Bacterial Fatty Acid Methyl Ester Extracts, Appl Environ Microb, 61, (1995) 1534. 
Figure captions

Figure 1. $\mathrm{CaO}$ reproducibility as a function of time. Square, E. coli; circle, $S$. typhimurium; triangle, S. boydii. Red, 0 hrs; green, 8 hrs; blue, 24 hrs.

Figure 2. Comparison of catalyst stability as a function of time. S. typhimurium spectra obtained over 24 hrs using A) $\mathrm{CaO}$ and $\mathrm{B}) \mathrm{CeO}_{2}$.

Figure 3. PCA plots of 10 bacteria from A) freshly activated $\mathrm{CaO}$ and $\mathrm{B}$ ) $\mathrm{Three}^{\mathrm{CeO}}$ time points. (о) A. baumannii, (о) B. anthracis, (о) C. putrefaciens, (о) E. coli, (•) E. faecalis, (০) F. tularensis, (০) L. monocytogenes, (०) S. aureus, (০) S. typhimurium, (o) Y.pestis.

Figure 4. Dendrogram of the Euclidean distances among the normalized spectra and the centroid averages.

Figure 5. FuRES classification tree built from the entire set of normalized spectra where $\mathrm{H}=$ entropy and $\mathrm{Nc}=$ number of objects in class. 


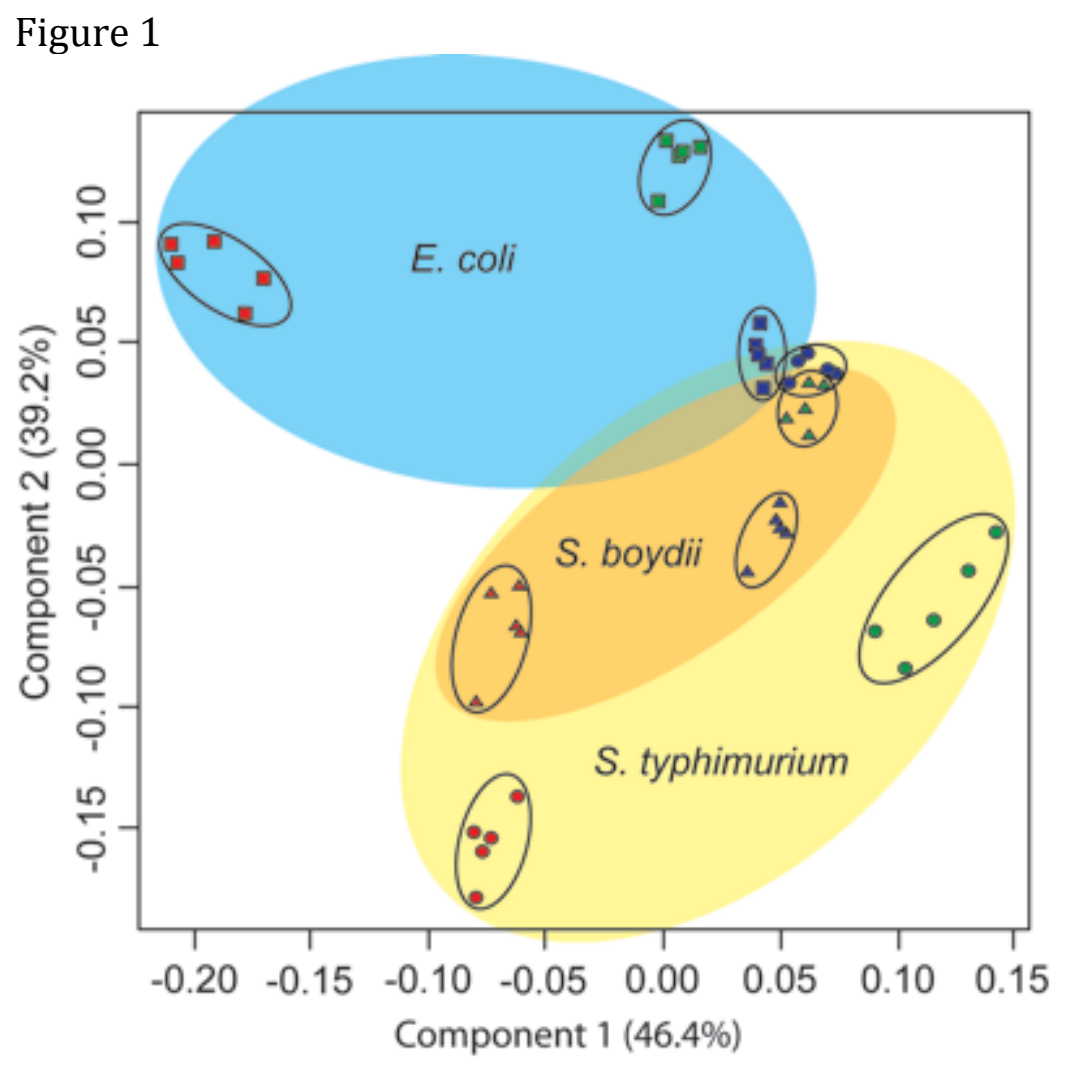

Figure 2
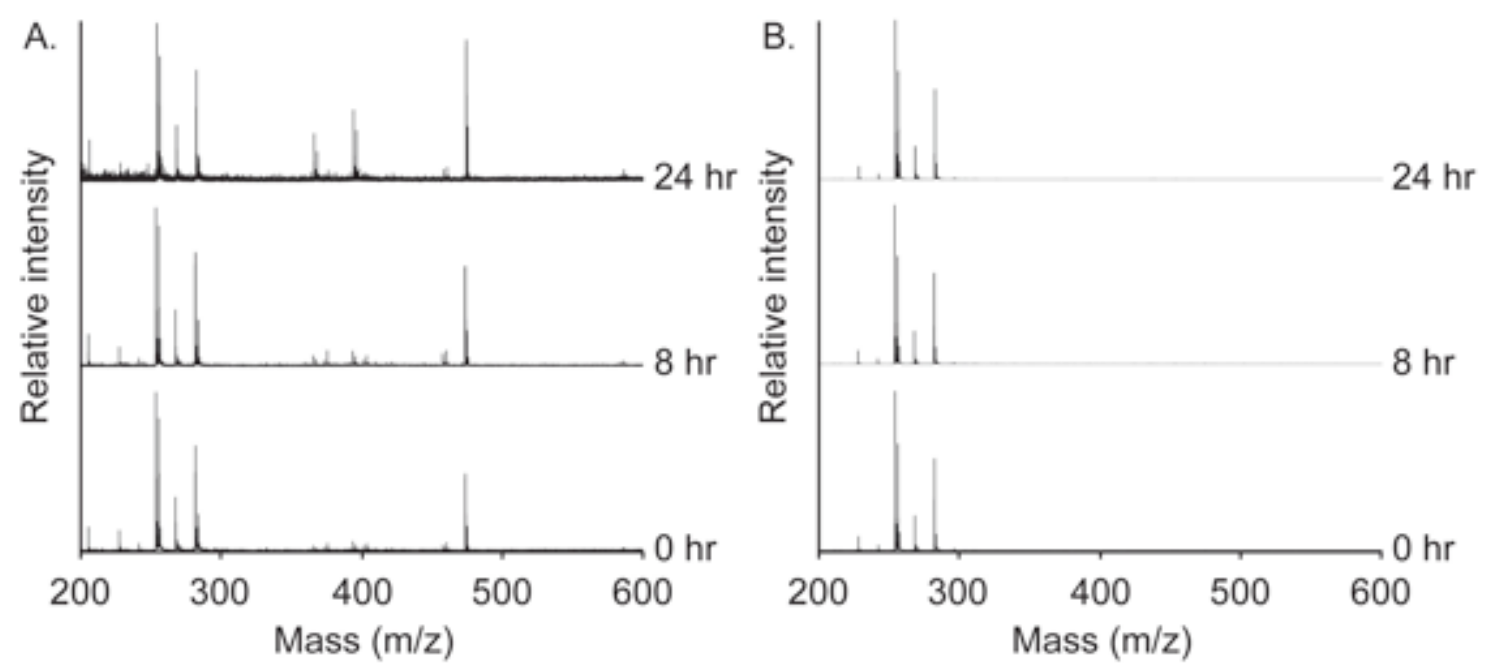
Figure 3
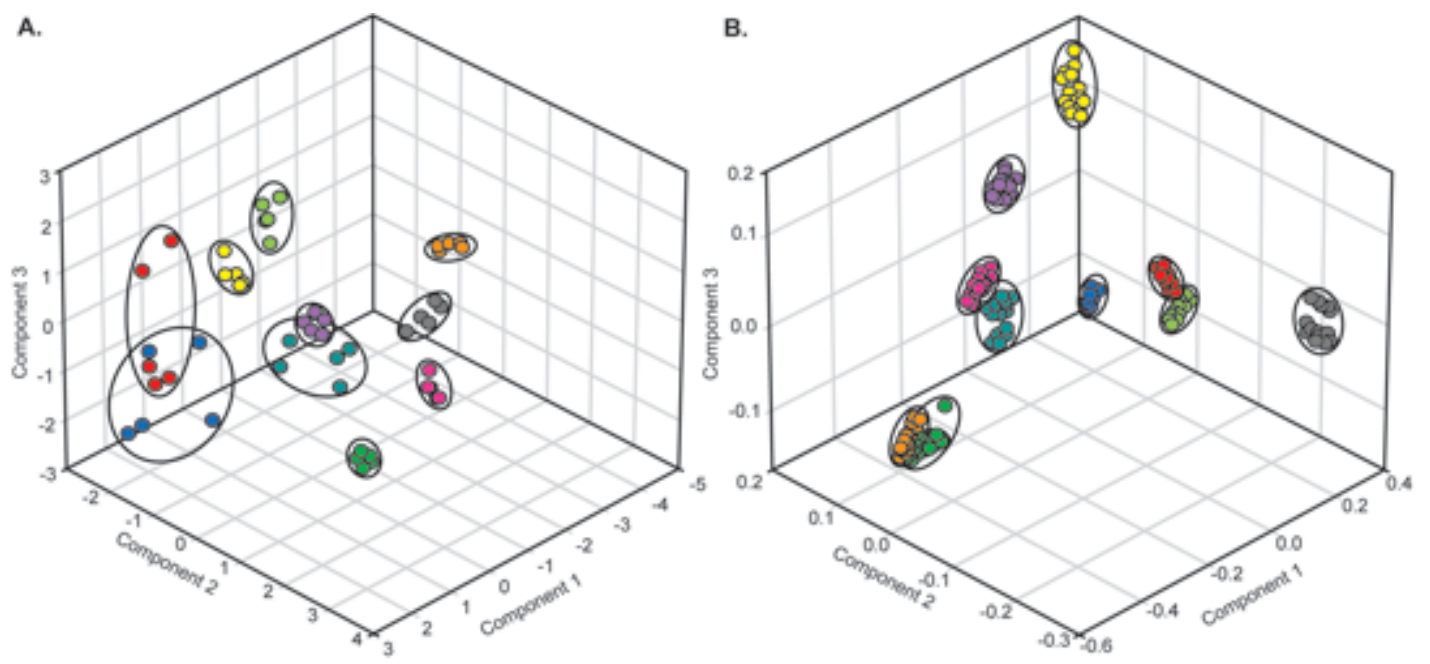
Figure 4

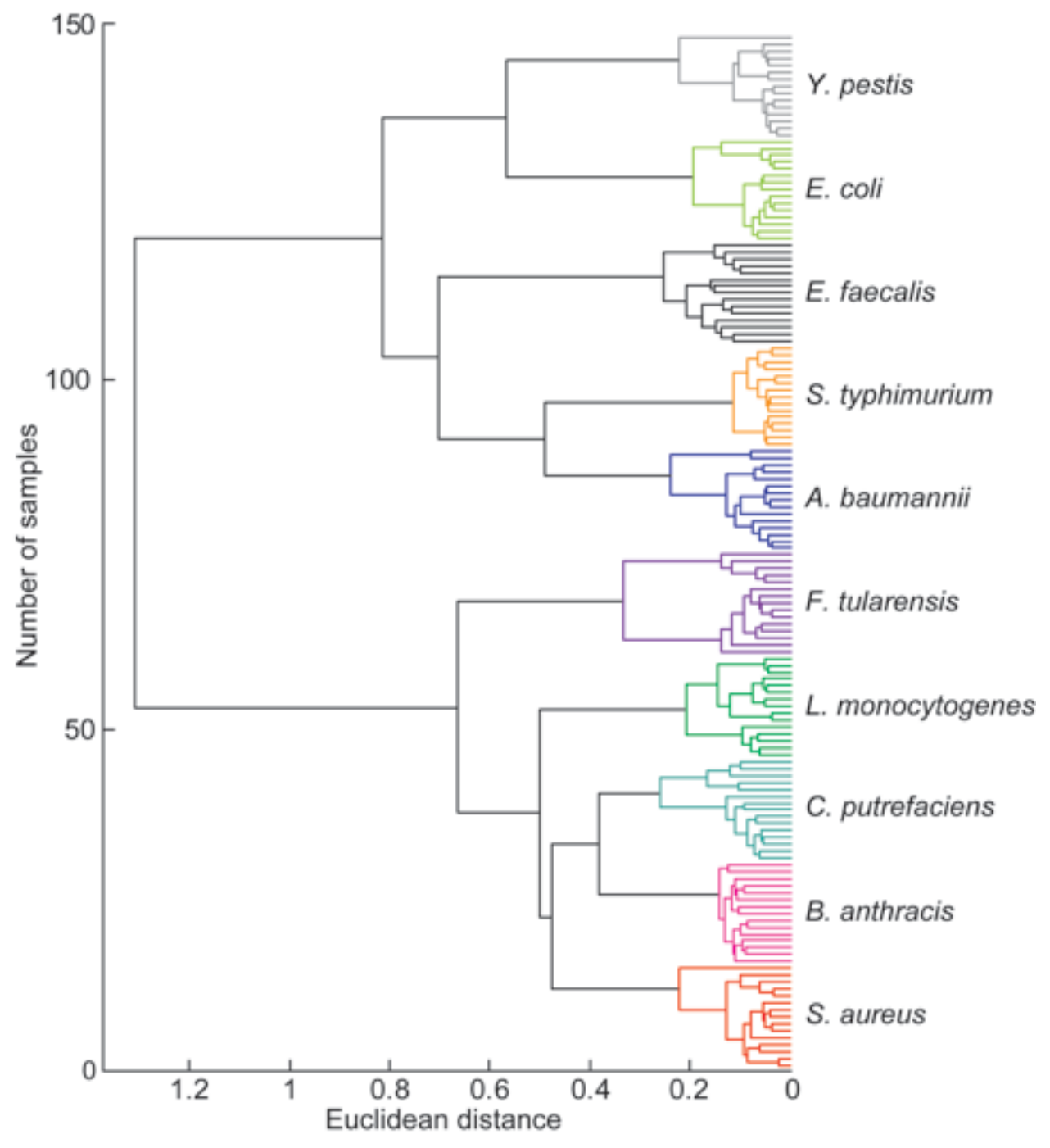


Figure 5

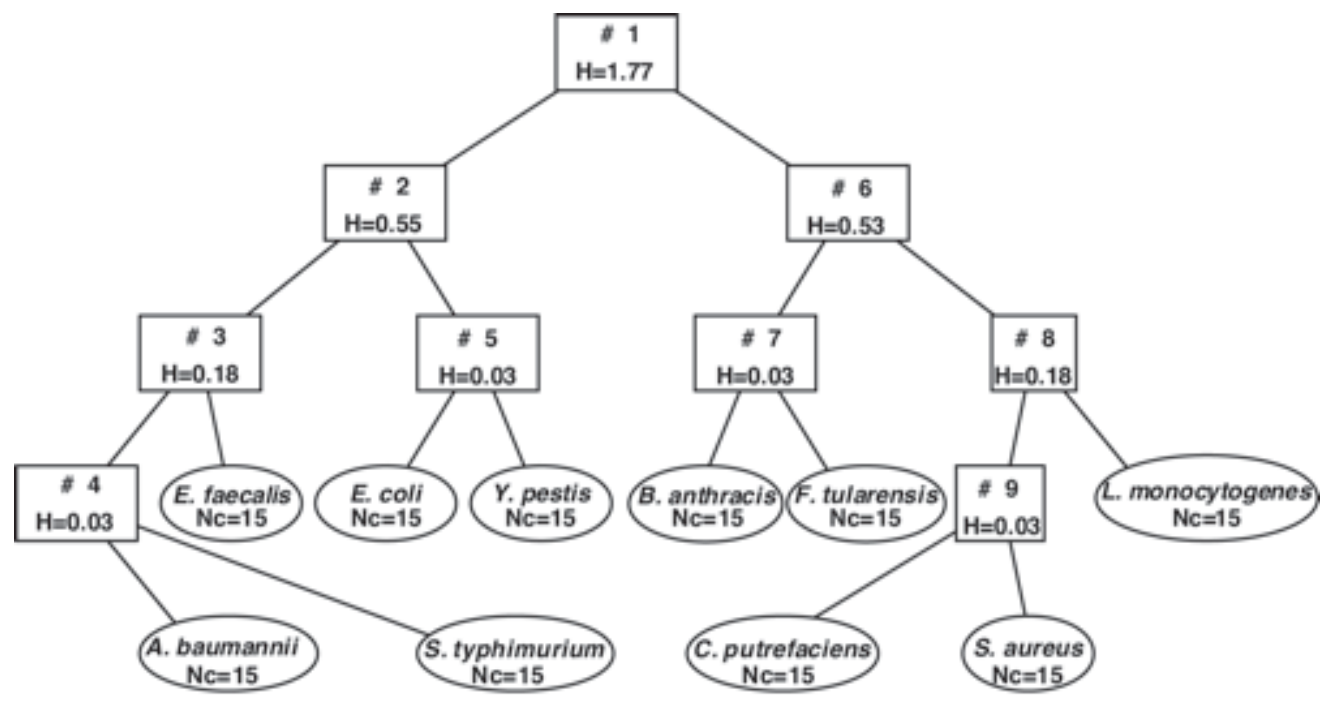


Table 1. Bacterial phylotypes used.

\begin{tabular}{lll}
\hline Gram positive & Strain & Source \\
\hline Bacillus anthracis & Sterne & Armed Forces Institute of Pathology \\
Clostridium putrefaciens & ATCC 25786 & ATCC \\
Enterococcus faecalis & V583 & University of Oklahoma Health Sciences Center \\
Listeria monocytogenes & ATCC 19112 & ATCC \\
Staphylococcus aureus & ATCC 27660 & ATCC \\
\hline Gram negative & & \\
\hline Acinetobacter baumannii & AC54 & Felix d'herelle Reference Center Viruses \\
Escherichia coli & ATCC 15597 & ATCC \\
Francisella tularensis & LVS & Colorado State University \\
Salmonella typhimurium & ATCC 13311 & ATCC \\
Yersinia pestis & A1122 & CDC Division of Vector-borne Diseases
\end{tabular}


Table 2. Fatty Acids used in Principal Component Analysis

\begin{tabular}{|c|c|}
\hline Fatty Acid & {$[\mathbf{M}-\mathbf{H}]^{-}$} \\
\hline C13:1 & 211 \\
\hline C13:0 & 213 \\
\hline C14:1 & 225 \\
\hline C14:0 & 227 \\
\hline C15:0 & 241 \\
\hline C16:1 & 253 \\
\hline C16:0 & 255 \\
\hline C17:2 & 265 \\
\hline C17:1 & 267 \\
\hline C17:0 & 269 \\
\hline C18:2 & 279 \\
\hline C18:1 & 281 \\
\hline C18:0 & 283 \\
\hline C19:1 & 295 \\
\hline C19:0 & 297 \\
\hline C20:1 & 309 \\
\hline C20:0 & 311 \\
\hline C21:1 & 323 \\
\hline C21:0 & 325 \\
\hline C22:1 & 337 \\
\hline C22:0 & 339 \\
\hline C23:1 & 351 \\
\hline C23:0 & 353 \\
\hline C24:1 & 365 \\
\hline C24:0 & 367 \\
\hline C25:1 & 379 \\
\hline C25:0 & 381 \\
\hline C26:1 & 393 \\
\hline C26:0 & 395 \\
\hline
\end{tabular}


Table 3. Percentage of total variance for $\mathrm{CaO}$ reproducibility

\begin{tabular}{c|c|c} 
Principal component & \% individual variance & \% cumulative variance \\
\hline 1 & 46.4 & 46.4 \\
3 & 39.2 & 85.6 \\
4 & 6.3 & 91.9 \\
5 & 5.9 & 97.9 \\
6 & .9 & 98.8 \\
& .7 & 99.5
\end{tabular}


Table 4. Comparison of percentage of total variance

\begin{tabular}{|c|c|c|c|c|c|}
\hline \multicolumn{3}{|c|}{$\mathrm{CeO}_{2}$} & \multicolumn{3}{|c|}{$\mathrm{CaO}$} \\
\hline $\begin{array}{c}\text { Principal } \\
\text { component }\end{array}$ & $\begin{array}{l}\text { \% individual } \\
\text { variance }\end{array}$ & $\begin{array}{c}\% \text { cumulative } \\
\text { variance }\end{array}$ & $\begin{array}{c}\text { Principal } \\
\text { component }\end{array}$ & $\begin{array}{c}\text { \% individual } \\
\text { variance }\end{array}$ & $\begin{array}{l}\text { \% cumulative } \\
\text { variance }\end{array}$ \\
\hline 1 & 82.7 & 82.7 & 1 & 20.7 & 20.7 \\
\hline 2 & 9.4 & 92.1 & 2 & 18.9 & 39.6 \\
\hline 3 & 5.7 & 97.8 & 3 & 11.3 & 50.6 \\
\hline 4 & 1.3 & 99.0 & 4 & 9.4 & 60.1 \\
\hline 5 & .6 & 99.6 & 5 & 7.9 & 68.2 \\
\hline 6 & .2 & 99.9 & 6 & 4.8 & 73.0 \\
\hline
\end{tabular}

